\title{
Combined Endoscopic/Microscopic Cochlear Implantation Through the Oval Window
}

\author{
Italo Cantore \\ Cochlear Implants Regional Center, San Carlo Regional Hospital, Potenza, Italy
}

Received June 23, 2021

Revised August 17,2021

Accepted September 16, 2021

\author{
Address for correspondence \\ Italo Cantore, $\mathrm{MD}, \mathrm{PhD}$ \\ Cochlear Implants Regional Center, \\ San Carlo Regional Hospital, \\ Via Potito Petrone snc, 85100 \\ Potenza, Italy \\ Tel +393889060901 \\ E-mail i.cantore@libero.it
}

Standard round window (RW) cochlear implantation is a well-described technique. Implantation might be difficult in patients with inner and middle ear anomalies, in some cases because of not achieving adequate exposure to the RW, with a related higher risk of complications such as facial nerve injury. It is proposed a combined microscopic/endoscopic oval window approach in a 63 year old man affected by bilateral Menière disease, with bilateral severe sensorineural hearing loss, speech discrimination score for bysillabic words under $40 \%$ and a hidden RW by anomalous facial nerve course. All electrodes entered the cochlear with good freefield thresholds and auditory ability results. A partial marginalis nerve palsy occurred at the second postoperative day and completely reversed at 2 months from surgery. Endoscopicassisted oval window cochlear implantation may be a safe alternative surgical technique in cases where surgeons are not able to access RW.

J Audiol Otol 2022;26(2):103-107

\section{Introduction}

Facial recess approach with cortical mastoidectomy, which was first introduced by House [1], is considered the gold standard surgical technique for cochlear implantation. This technique has been used worldwide, with good safety and otologic surgeons compliance. However, it is sometimes difficult to access the round window (RW) and promontory via posterior tympanotomy in cases of anatomic diversities, such as an anomalous facial nerve $(\mathrm{FN})$ course, narrow facial recess, low middle fossa dura, anterior sigmoid sinus. Among them, FN damaging and consequent paralysis are the main fearsome point, especially for patients with inner-ear or FN malformations [2]. Although otologists routinely evaluate aberration of the FN using high-resolution temporal bone computed tomography or cone beam computed tomography (CBCT) and with these methods it is almost always possible to follow the course of the FN if carefully evaluated, in very few particular cases this cannot be identified completely [3]. Some inner ear malformations may cause aberrations in the location of the FN ca-

This is an Open Access article distributed under the terms of the Creative Commons Attribution Non-Commercial License (https://creativecommons.org/licenses/by-nc/4.0/) which permits unrestricted non-commercial use, distribution, and reproduction in any medium, provided the original work is properly cited. nal, making its course more complicated [4]. These aberrations can impede electrode implantation via a routine RW approach and increase the risk of iatrogenic FN injury [5]

In order to safely address some difficult selected cases, various alternative surgical approaches were proposed, combining solo microscopic ways [6-8], and, more recently, endoscopic/ microscopic combined ones [9-11]. Still, in some cases, RW access is really difficult even with these combined approaches.

\section{Case Report}

A 63-year-old man, affected by bilateral Menière disease, with bilateral severe sensorineural hearing loss, high recruitment and speech discrimination score for bysillabic words under 40\%, underwent cochlear implant candidacy evaluation protocol at our center. Preoperative CBCT (Fig. 1) and MRI, showed cochlear patency, no evidence of second intratympanic facial tract under lateral semicircular canal on coronal CBCT reconstruction and the suspect of both thickened RW bony wall and inferior course of FN.

The patient gave informed consent concerning the possibility of alternative surgical techniques, followed by a left cochlear implant surgery, under FN monitoring, by means of a classical masthoidectomy, incus short process localization 
and posterior tympanotomy. Due to the evidence of oval windows (OW) uncomplete visualization, absence of second intratympanic tract of FN prominence over it, and an anomalous inferior FN tract, with anteriorization of stapedius tendon eminence that completely hid the RW, evaluated as a type III St. Thomas OW exposure stage [12], in order to have better viewings it was first tried incus removal, tympanomeatal flap preparation and a combined endomeatal approach [8], with few results in terms of oval window visualization. It was then explored endoscopically the medial tympanic wall, through both endomeatal and facial recess ways, by means of a $14 \mathrm{~mm}$ length, 3-mm diameter, $0^{\circ}$ and $45^{\circ}$ endoscopes mounted on his HD dedicated camera (Karl Storz, Tuttlingen, Germany).
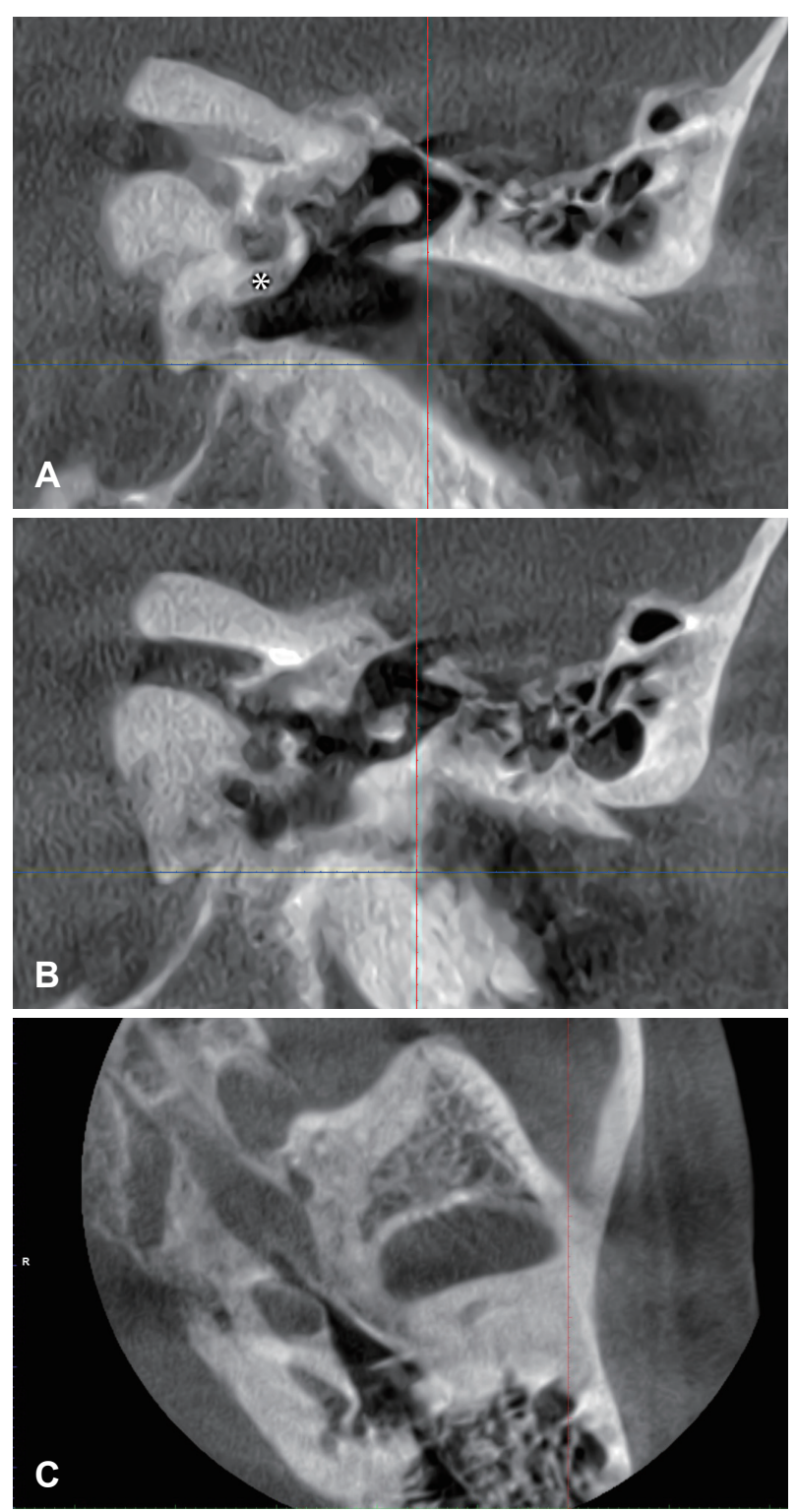

Fig. 1. Preoperative coronal (A and $B$ ) and axial $(C)$ cone beam computed tomography reconstructions. Images suggest thick round window bony wall and inferior course of facial nerve $\left(^{*}\right)$.
Nevertheless, the anomalous FN course did not allow access to the cochlea through RW membrane or traditional cochleostomy, and furthermore it limited the exposure of the OW. Due to the uncomplete $\mathrm{OW}$ visualization even after stapes removal under microscopic view and the FN inferior abnormal course, it was decided to make array insertion through OW under combined endoscopic and microscopic control (Fig. 2) using a Cochlear CI512 implant (Cochlear Ltd., Sydney, Australia) with his rigid stylet linked configuration which should facilitate the avoidance of the lateral semicircular canal way. Access to the OW, after stapes removal, and electrode array insertion starting were performed under endoscope viewing, in order to better control the partial exposure. Electrode array progression inside the cochlea was completed under microscope control. The presence of normal neural response telemetry values on all electrodes confirmed the correct and complete intracochlear electrode array positioning, with no needing to perform intraoperative imaging in order to exclude lateral semicircular canal entering.

After 24 hours from surgery the patient showed a progressive unilateral incomplete left House-Brackmann stage III facial palsy, interesting mainly mimic muscles of marginalis nerve pertinence, with slight involvement of cervical-facial branch, related to FN canal wall drilling across its anomalous course in correspondence of the RW site, and subsequent edema. It was started an intravenous therapy with metilprednisolone 40 mg 1 bottle two times per day for 3 days, then 1 bottle one time per day for 7 days, subsequently $16 \mathrm{mg} 1$ capsule per day for 5 days, and $1 / 2$ capsule per day for the following 4 days. One month after surgery a partial palsy of the left marginalis nerve innervated mimic muscles persisted; after one further month, no palsy was detectable (House-Brackmann stage I). No other relevant symptoms, as dizziness or tinnitus enhancement, were reported after surgery. Post-operative CBCT (Fig. 3) showed correct and complete intracochlear array positioning (Fig. 4).

Implant was activated after 15 days from surgery with a Kanso 2 processor (Cochlear Ltd.) programmed in ACE and scan mode with progressive fitting implementations and adult speech therapy rehabilitation protocol. Three months after surgery, the patient showed very good free-field thresholds (20-35 dB HL for pure tones) and nice ongoing auditory abilities improvements $(70 \%$ recognition scores for bysillabic words, $80 \%$ comprehension).

The informed consent was obtained from the participant.

\section{Discussion}

Alternative surgical techniques to traditional posterior 
tympanotomy have been described over the last years, mainly in order to have a better medial tympanic wall visualization and to reduce risks of FN injury, which is the most concerning complication due to bony overhangs or an abnormal course of the nerve. The ratio of facial paralysis following cochlear im-
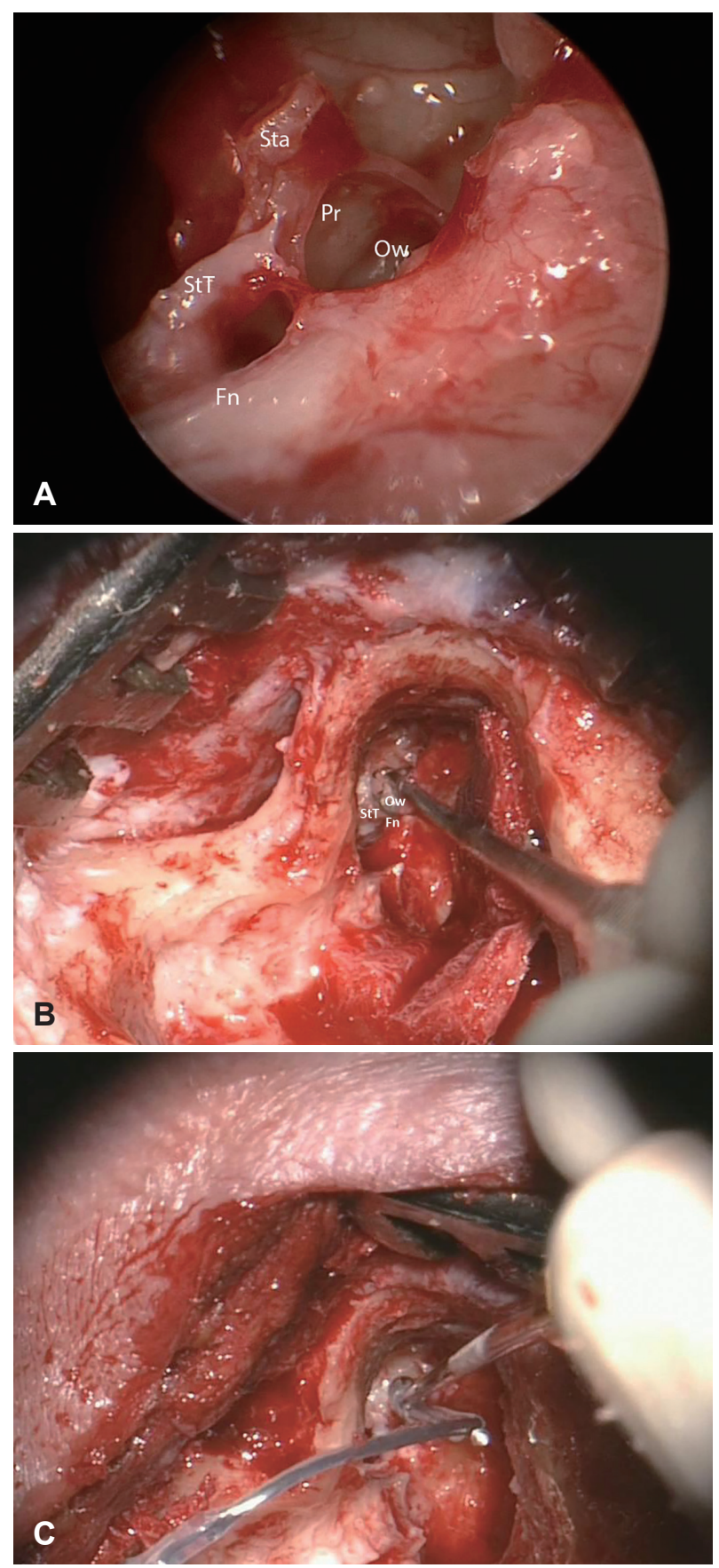

Fig. 2. Intraoperative endoscopic view (A) and microscopic ones $(B$ and $C)$. The endoscope was very useful in order to clarify the complete round window inaccessibility, to better view the oval window, partially hidden, to proceed to its incision and to start electrode array insertion, which was completed by means of microscopic control. StT, stapedius tendon; Fn, facial nerve; Sta, stapes; Pr, promontory; Ow, oval window (left ear). plantation has been reported as $0.07 \%-1.1 \%$ in literature, and most of these reported cases were delayed onset paralysis [3].

Kronenberg, et al. [6] developed the suprameatal route that avoids mastoidectomy and allows introduction of the electrode into the middle ear via a suprameatal approach, in this case the FN is protected by the incus; the disadvantages of this technique are: the presence of low-lying dura; stretching of the electrodes during insertion into the cochleostomy; difficult
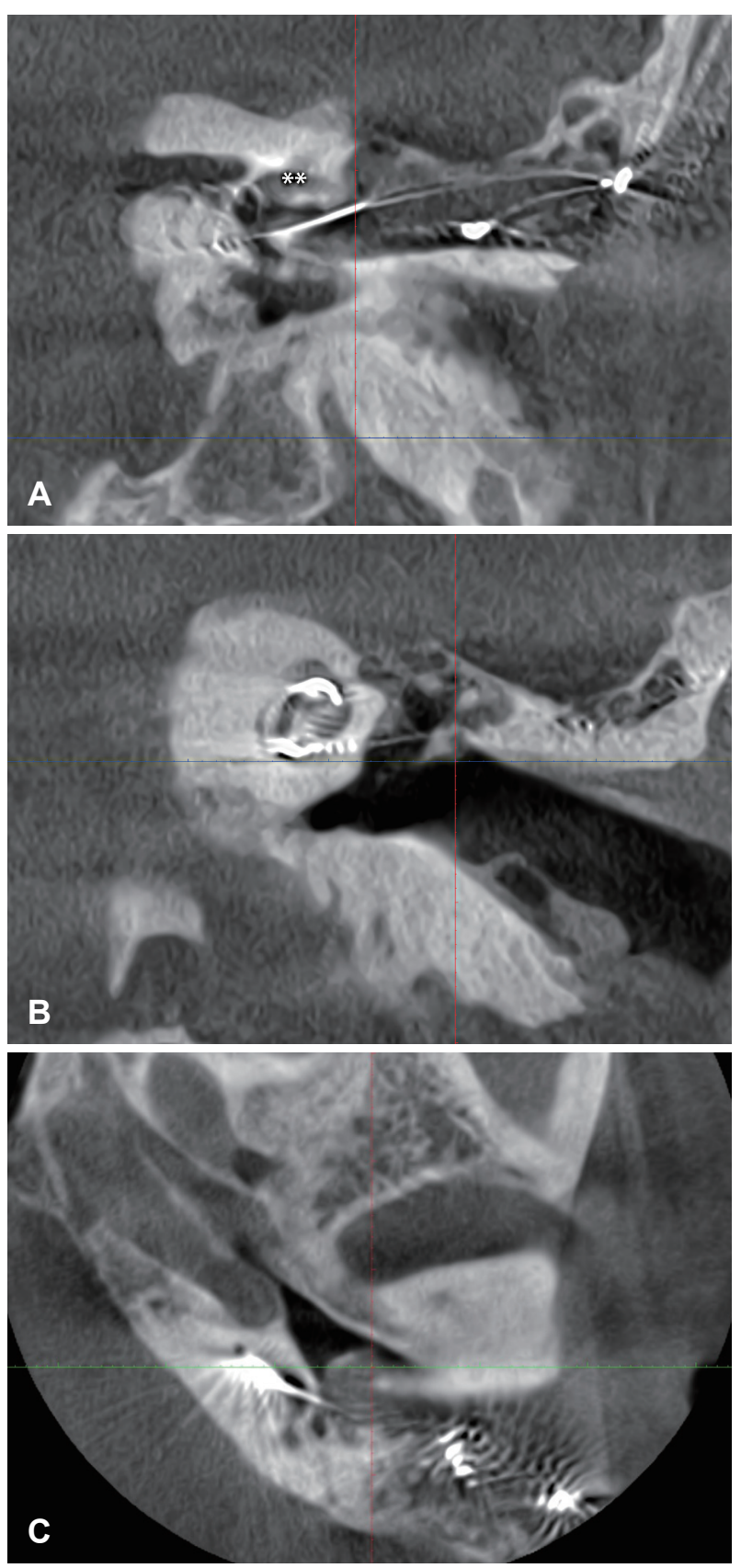

Fig. 3. Postoperative coronal ( $A$ and $B$ ) and axial $(C)$ cone beam computed tomography reconstructions. It is shown the electrode array entering through the oval window, its correct and complete electrode array insertion inside the cochlea. ${ }^{* *}$, lateral semicircular canal. 


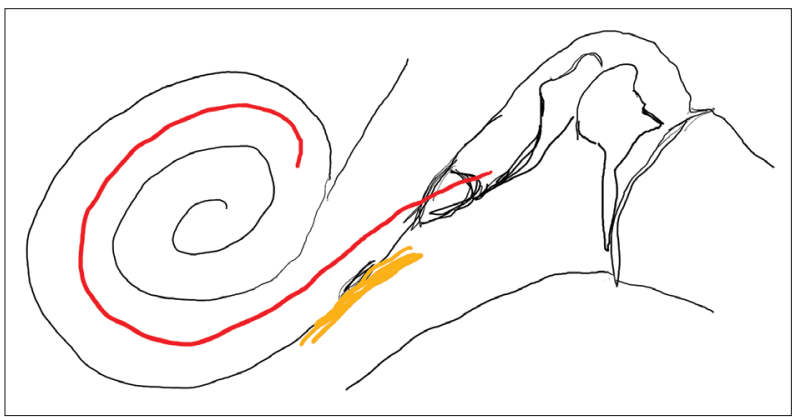

Fig. 4. A schematic drawing of abnormal facial nerve course (yellow colour) and the electrode array placing through the oval window (red colour).

RW visualization and insertion, with potentially difficult inferior cochleostomy. As an alternative to this suprameatal alone way it was proposed the "Veria" operation [7]. It is a non-mastoidectomy approach, and uses an endaural approach for the cochleostomy and a supero-posterior approach for the electrode. It allows better medial tympanic wall visualization, but with the disadvantage of requiring specialized instrumentation and reduced operative space. A combined microscopic access way which includes endomeatal and posterior tympanotomy was proposed Della Volpe, et al. [8] in 2013, with better results in terms of complete electrode array insertion in comparison with posterior tympanotomy alone. In this combined approach technique, a transcanal approach cochleostomy and array insertion control is combined with a small posterior tympanotomy for positioning the electrode array inside the tympanic cavity and its insertion inside the cochlea.

The diffusion of endoscopic otologic surgery techniques and instruments over last years conducted to very useful and innovative combined endoscopic/microscopic techniques with main benefit of improving visibility leading to a panoramic view of the RW region. Dia, et al. [13] reported an endoscopic cochlear implantation surgery with an endoscopic transcanal electrode insertion without mastoidectomy, covering electrode by means of a a fascial graft, with almost 1 case on 4 of electrode extrusion and higher infection risk. Marchioni, et al. [9] reported also in 2014 an endoscopic transcanal technique, but they created a small bone groove on the facial recess and also created an intramastoid tunnel starting from Henle spine area, parallel to the external ear, and connected to the bony groove. They inserted the electrodes through this tunnel to avoid the complications, which seems to be safer in terms of extrusion possibility. Migirov, et al. [14] introduced a combined endoscopic/microscopic technique with RW visualization using an endoscope through the external ear canal and inserting the electrode through a tunnel drilled with microscope from the mastoid to the epitympanum. Orhan, et al. [10] introduced endoscope through the facial recess, after a traditional microscope posterior tympanotomy, in order to better visualize RW niche and proceed to its drilling for cochleostomy through endomeatal way under endoscopic trans-facial recess control. As an alternative combined endoscopic/microscopic technique, more recently it was proposed to proceed endoscopically through meatal way by means of tympanomeatal flap elevation, in association with a microscopic step via transmastoid transattical route [11], with no complications and improvement in terms of RW visualization.

In those cases of RW not only hidden but even not accessible due to anatomic variations, an alternative way to enter the cochlea has to be evaluated. The OW access way is a possibility in such cases, with the disadvantages that this surgical approach may cause electrode array entry through the vestibule and semicircular canal only, and that electrode array have to bend over in order to access properly the cochlea, with consequent possibility of tip folding or electrodes damage. Furthermore, apart this wrong way access inside vestibule and semicircular canal, vestibule damaging and post-surgical dizziness possibility has ever to be considered as a concrete possibility when this surgical method is selected. In order to limit such complications possibilities and obtain better electrostimulation conditions, in those cases without the possibility of scala tympani access, which has ever to be preferred, it should be considered the possibility to enter scala vestibuli in its second turn. Chen, et al. [15] in a pediatric case with abnormal FN inferior course analogous to Author's reported one, performed incus and stapes removal through facial recess microscopic direct view and entered the OW without the possibility of a wider and more detailed endoscopic view of inferior portions of tympanic medial wall. They reported good audiologic results but with 2 electrodes positioned into the vestibule. Combining traditional posterior tympanotomy microscopic access with endoscopic one even via transmeatal way should, in similar cases, allows better tympanic medial wall exposure and facilitates array inserting, regardless of the accessibility of the RW.

In conclusion, combined endoscopic/microscopic cochlear implant surgery is a safe procedure and can improve tympanic medial wall visualization and cochlear access even in anatomical variants such as FN course anomalies. Despite its possible complications, OW array entering under endoscopic control can be considered in those cases with hidden/inaccessible RW and unsafe cochleostomy.

\section{Acknowledgments}

Thanks to the passion of taking care of deaf people transmitted by Dr. Rocco Cantore. And to precious teachings of Prof. Gaetano Paludetti, Prof. Walter Di Nardo, Dr. Antonio Della Volpe, Prof. Thomas Balkany and Prof. Thomas Roland Jr. 


\section{Conflicts of interest}

The author has no financial conflicts of interest.

\section{ORCID iD}

Italo Cantore

https://orcid.org/0000-0002-3860-7663

\section{REFERENCES}

1) House WF. Cochlear implants. Ann OTOL Rhinol Laryngol 1976; 85:1-93.

2) Sennaroglu L. Cochlear implantation in inner ear malformations-a review article. Cochlear Implants Int 2010;11:4-41.

3) Kashio A, Sakamoto T, Karino S, Kakigi A, Iwasaki S, Yamasoba T. Predicting round window niche visibility via the facial recess using high-resolution computed tomography. Otol Neurotol 2015; 36:18-23.

4) Song JJ, Park JH, Jang JH, Lee JH, Oh SH. Chang SO, et al. Facial nerve aberrations encountered during cochlear implantation. Acta Otolaryngol 2012;132: 788-94.

5) Huang CC, Lin CY, Wu JL. Retrofacial approach of cochlear implantation in inner ear malformation with aberrant facial nerve: a case report. Auris Nasus Larynx 2006;33:179-82.

6) Kronenberg J, Migirov L, Baumgartner WD. The suprameatal approach in cochlear implant surgery: our experience with 80 patients. ORL J Otorhinolaryngol Relat Spec 2002;64:403-5.

7) Kiratzidis T, Iliades T, Arnold W. Veria operation. II. Surgical re- sults from 101 cases. ORL J Otorhinolaryngol Relat Spec 2002;64: 413-6.

8) Della Volpe A, Cantore I, Nolè G, Valente P, Varricchio AM, Santandrea M, et al. Combined posterior tympanotomy/endomeatal access in cochlear implantation. Eur Arch Otorhinolaryngol 2013; 270:2641-8.

9) Marchioni D, Grammatica A, Alicandri-Ciufelli M, Genovese E, Presutti L. Endoscopic cochlear implant procedure. Eur Arch Otorhinolaryngol 2014;271:959-66.

10) Orhan KS, Polat B, Çelik M, Çomoğlu S, Güldiken Y. Endoscopicassisted cochlear implantation: a case series. J Int Adv Otol 2016; 12:337-40.

11) Carner M, Sacchetto A, Bianconi L, Soloperto D, Sacchetto L, Presutti L, et al. Endoscopic-assisted cochlear implantation in children with malformed ears. Otolaryngol Head Neck Surg 2019;161: 688-93.

12) Leong AC, Jiang D, Agger A, Fitzgerald-O'Connor A. Evaluation of round window accessibility to cochlear implant insertion. Eur Arch Otorhinolaryngol 2013;270:1237-42.

13) Dia A, Nogueira JF, O'Grady KM, Redleaf M. Report of endoscopic cochlear implantation. Otol Neurotol 2014;35:1755-8.

14) Migirov L, Shapira Y, Wolf M. The feasibility of endoscopic transcanal approach for insertion of various cochlear electrodes: a pilot study. Eur Arch Otorhinolaryngol 2015;272:1637-41.

15) Chen B, Li Y, Lu S, Chen J, Shi Y, Cui D, et al. Cochlear implant surgery through oval window: a novel approach in a child with facial nerve aberration. Int J Pediatr Otorhinolaryngol 2020;135:110110. 